\title{
Prevalência de sintomas de estresse nos estudantes de medicina
}

\author{
Prevalence of stress symptoms among medical students
}

Sâmia Mustafa Aguiar', Anya Pimentel Gomes Fernandes Vieira², Karine Magalhães Fernandes Vieira', Sabrine Mustafa Aguiar ${ }^{3}$, Joana Oliveira Nóbrega ${ }^{3}$

\section{RESUMO}

Objetivo: Identificar o perfil sociodemográfico dos acadêmicos de Medicina e avaliar a prevalência de sintomas de estresse nesses estudantes. Métodos: A população estudada respondeu ao Inventário de Sintomas de Stress para Adultos de Lipp (ISSL), para identificação de sintomas de estresse e a um questionário sociodemográfico. Resultados: Duzentos estudantes de Medicina, matriculados nos Ciclos Básico ou Clínico da Universidade Federal do Ceará (UFC), foram estudados. Houve predominância do sexo masculino $(54,5 \%)$, solteiros (100\%), naturais de Fortaleza (87\%), com idade média de 21 ( $\pm 2,3)$ anos. As alunas apresentaram níveis de sintomas de estresse maiores do que os estudantes homens, representando 30,1\% e 19,6\%, respectivamente ( $p \leq 0,001)$. A prevalência de sintomas de estresse foi de $49,7 \%$, sendo o quarto semestre o período com níveis mais altos desses sintomas $(p=0,002)$. Conclusões: Conclui-se que estudantes de Medicina da Universidade Federal do Ceará (UFC) têm níveis de estresse similares àqueles relatados para amostras internacionais.

\section{Palavras-chave}

Estresse, estudantes de Medicina.

\section{Keywords}

Stress, medical students.

\section{ABSTRACT}

Objective: To study the social-demographic profile of Medical students and assess the prevalence of stress symptoms in these students. Methods: The Inventory of Symptoms of Stress for Adults of Lipp (ISSL) was used to evaluate the symptoms of stress and a questionnaire was applied to identify the participants' social-demographic characteristics. Results: Two hundred Medical students registered at the Federal University of Ceará (UFC), taking Basic and Clinical courses, were interviewed. Participants were predominantly male (54.5\%), single (100\%), born in Fortaleza (87\%), mean age 21 years $(S D=2.3)$. Female students showed higher levels of stress' symptoms than male students, $30.1 \%$ and $19.6 \%$ respectively, $(p \leq 0.001)$. The prevalence of symptoms of stress was $49.7 \%$ and students attending the fourth semester showed the highest levels of symptoms ( $p=0.002)$. Conclusions: The conclusion is that Medical students at UFC have levels of stress similar to those reported in international studies.

1 Universidade de Fortaleza (Unifor).

2 University of Toronto, Canadá. 


\section{INTRODUÇÃO}

A saúde mental dos profissionais de saúde constitui motivo de preocupação desde o início do século, tendo em vista o caráter estressante do trabalho em saúde. A natureza do exercício profissional e da organização desse trabalho pode concorrer para o desenvolvimento de distúrbios emocionais?.

O médico tem sido objeto frequente de estudos psicológicos e sociológicos, tendo em vista a natureza e as repercussões singulares de sua atividade. A carreira médica representa, ainda hoje, a possibilidade de satisfação para o indivíduo, dos anseios materiais, emocionais e intelectuais². Apesar disso, estudos apontam para uma prevalência elevada do uso de drogas, depressão, suicídio, distúrbios conjugais e disfunções profissionais em médicos e acadêmicos de medicina ${ }^{3,4}$.

A literatura descreve vários momentos potencialmente estressantes na vida do acadêmico de Medicina, sendo a formação e a atividade médica consideradas de elevada toxicidade no tocante ao aspecto psicológico, e aponta vários motivos para o estresse entre os estudantes de Medicina, que também são encontrados em alguns outros cursos da área de saúde ${ }^{5-10}$. O vestibular extremamente competitivo, a metodologia de ensino que é diferente da usada no colegial, o curso básico longo que adia o contato com a profissão propriamente dita e pode ocasionar frustração ao aluno, o ritmo de plantões e a escolha da especialidade destacam-se como fatores de estresse. Adicionalmente, alguns momentos do curso, como o primeiro contato com o paciente e o término da faculdade, podem ser críticos para o estudante, quando o receio de sua atuação como profissional de saúde pode emergir.

Acrescentam-se a isso as situações em que o aluno reside sozinho e distante de casa, o período longo e em tempo integral dos cursos, a grande quantidade de informações que precisa adquirir, a qualidade da relação professor-aluno e a influência da atividade acadêmica sobre suas atividades de lazer e relacionamentos sociais. Além disso, após a conclusão do curso, o ex-estudante de medicina enfrenta um exame cada vez mais competitivo para ingresso em um programa de residência médica ou o ingresso imediato no mercado de trabalho, sem mais a retaguarda da escola ${ }^{5,11,12}$.

Como visto, o contato com doença grave, sofrimento e morte pode representar importante fonte de estresse já no período de treinamento do estudante de medicina, desde o modelo tradicional das faculdades, com enfoque racional, não emocional e científico, contribui para o despreparo do aluno diante dessas situações ${ }^{5,7,13}$.

Este estudo teve como objetivos identificar o perfil sociodemográfico dos acadêmicos de Medicina e avaliar a prevalência de sintomas de estresse nesses estudantes, visto que a atenção à saúde desses futuros profissionais pode repercutir no seu bem-estar e da população por eles assistida.

\section{MÉTODOS}

O desenho do presente estudo foi descritivo e transversal. Com o objetivo de avaliar a prevalência da sintomatologia de estresse nos diferentes momentos do curso de Medicina, foram considerados, nesse estudo, o $1^{\circ}$ semestre, por ser o momento de ingresso na faculdade, $04^{\circ}$ semestre, por ser o início do treinamento clínico e o $8^{\circ}$ semestre, que antecede a entrada no internato. A amostra incluiu 200 estudantes, regularmente matriculados, cursando pelo menos $70 \%$ dos créditos previstos para o semestre e não repetentes. O estudo foi realizado na Faculdade de Medicina da Universidade Federal do Ceará (UFC).

A coleta dos dados foi realizada em março de 2007. Os questionários foram aplicados nas salas de aulas, com permissão prévia dos professores. Após terem sido esclarecidos sobre os métodos da pesquisa, aqueles que concordaram em participar assinaram um termo de consentimento livre e esclarecido. A aplicação foi coletiva e os instrumentos eram autorrespondidos e consistiram de um Questionário Sociodemográfico e do Inventário de Sintomas de Stress para Adultos de Lipp ${ }^{14}$. O projeto foi aprovado pelo Comitê de Ética em Pesquisa da Universidade Federal do Ceará (UFC), onde foi realizado o estudo (município de Fortaleza).

O Inventário de Sintomas de Stress para Adultos de Lipp (ISSL) foi validado no Brasil em 1994 por Lipp e Guevara e tem sido utilizado em pesquisas e trabalhos clínicos na área do estresse $\mathrm{e}^{15,16}$, permitindo um diagnóstico claro da existência de sintomas de estresse, bem como da fase em que se encontra (alerta, resistência, quase exaustão e exaustão) e da sintomatologia predominante, se física ou psicológica. O ISSL apresenta um modelo quadrifásico do estresse acerca das suas manifestações nas esferas somática e cognitiva, da sequência e gradação de seriedade dos sintomas. A sua aplicação pode ser feita por pessoas que não tenham treinamento em psicologia, mas a análise deve ser sempre realizada por um psicólogo, em cumprimento às diretrizes do Conselho Federal de Psicologia'7. Nesse estudo, a análise foi realizada pela psicóloga coautora do trabalho (KMFV).

Os dados foram tabulados no SPSS - Statistical Package for the Social Science (SPSS Inc., Chicago, IL, USA), versão 13.0 para Windows, agrupados em tabelas e analisados de acordo com os objetivos da pesquisa. Quando comparados os resultados categóricos entre subgrupos, o teste Qui-quadrado foi utilizado.

\section{RESULTADOS}

Participaram do estudo 88,9\% $(n=200)$ dos 225 acadêmicos matriculados no período. Desses, $99,5 \%(n=199)$ responderam ao ISSL. 
$\mathrm{Na}$ amostra estudada, verificou-se predomínio de sexo masculino (54,5\%). A média de idade encontrada foi de 22 anos, com um desvio-padrão de 2,3 anos. Quanto ao estado civil, $100 \%$ eram solteiros e $85,3 \%(n=169)$ moravam com os pais dos 198 (98\%) alunos que responderam a essa questão. Dos 197 (98,5\%) acadêmicos que responderam a essa pergunta, $85,3 \%(n=168)$ informaram não trabalhar e ter seus gastos financiados pela família. Com relação à conclusão do ensino médio, dos 197 (98,5\%) que responderam a essa questão, a maioria dos acadêmicos $(93,4 \%)$ terminou em escola privada.

As amostras referentes aos primeiro, quarto e oitavo semestres compreenderam, respectivamente, $87,2 \%(n=68)$, $87 \%(n=67)$ e $92,8 \%(n=65)$ dos acadêmicos regularmente matriculados nesses semestres. A idade média era de 19,1 \pm $1,8,21,2 \pm 1,7$ e $22,8 \pm 1,7$ anos para o $1^{\circ}, 4^{\circ}$ e $8^{\circ}$ semestres, respectivamente.

A maior parte dos acadêmicos do $1^{\circ}$ semestre referiu não desenvolver atividade acadêmica além das obrigatórias $(92,6 \%)$, nem realizar estágio acadêmico em regime de plantões não obrigatórios (98,5\%). Já no $4^{\circ}$ e $8^{\circ}$ semestres, $68,7 \%(n=46)$ e $98,5 \%(n=64)$ dos alunos, respectivamente, participavam de atividade acadêmica além das obrigatórias, apesar de a maioria dos estudantes do 40 semestre (91\%) não realizar estágio acadêmico em regime de plantões em contrapartida a $75,4 \%(n=49)$ dos acadêmicos do $8^{\circ}$ semestre, que participavam de plantões não obrigatórios. Embora $71 \%(n=142)$ de todos os estudantes informassem jornada de sono acima de 6 horas habitualmente, por noite, e 55\% ( $n=110$ ) considerarem sua qualidade de sono melhor do que se possa imaginar, mais da metade $(65,5 \%)$ dos estudantes relataram não se sentir bem repousados ao acordar pela manhã.

Dos 200 estudantes que participaram do estudo, 73,5\% $(n=147)$ consideraram suas atividades na faculdade como fonte de estresse. Apesar dessa percepção, apenas 35,8\% $(n=71)$ informaram realizar alguma atividade para a promoção de saúde física e/ou mental dos 198 (99\%) alunos que responderam a essa pergunta.

A prevalência de sintomas de estresse nos estudantes de Medicina foi de 49,7\% ( $n=99)$. Quando estratificada por semestres, a população mostrou comportamento diferenciado quanto à ocorrência de estresse, porém não houve diferença entre as fases do estresse e os sintomas predominantes entre os três semestres avaliados, como apresentado na tabela 1. Vale ressaltar a predominância dos sintomas de estresse na fase de resistência, bem como dos sintomas na esfera psicológica nos três semestres avaliados. Além disso, a ocorrência de estresse nos acadêmicos, em geral, foi maior no sexo feminino $(66,7 \%)$ do que no masculino $(35,8 \%)$, com $p \leq 0,001$.

Dos acadêmicos com sintomas de estresse, 85,8\% $(n=85)$ encontravam-se na fase de resistência, enquanto
Tabela 1. Comparação da prevalência de estresse, fases e sintomas predominantes de estresse nos acadêmicos de Medicina do 1ㄴ, 4은 e 8o semestres

\begin{tabular}{|c|c|c|c|c|c|c|c|}
\hline & \multicolumn{7}{|c|}{ Semestre } \\
\hline & \multicolumn{2}{|c|}{$1^{0}$} & \multicolumn{2}{|c|}{$4^{0}$} & \multicolumn{2}{|c|}{$8^{\circ}$} & \multirow[t]{2}{*}{$p$} \\
\hline & $n$ & $\%$ & $n$ & $\%$ & $\mathrm{n}$ & $\%$ & \\
\hline Estresse & 28 & 41,2 & 45 & 67,2 & 26 & 40,6 & 0,002 \\
\hline \multicolumn{8}{|l|}{ Fase do estresse } \\
\hline Alerta & 3 & 10,7 & 3 & 6,7 & 3 & 11,5 & \multirow[t]{4}{*}{0,792} \\
\hline Resistência & 23 & 82,1 & 40 & 88,9 & 22 & 84,6 & \\
\hline Quase-exaustão & 1 & 3,6 & - & - & - & - & \\
\hline Exaustão & 1 & 3,6 & 2 & 4,4 & 1 & 3,8 & \\
\hline \multicolumn{8}{|l|}{ Sintomas predominantes } \\
\hline Psicológicos & 19 & 67,8 & 32 & 71,1 & 17 & 68 & \multirow[t]{3}{*}{0,597} \\
\hline Físicos & 5 & 17,9 & 8 & 17,8 & 7 & 28 & \\
\hline Psicológicos e físicos & 4 & 14,3 & 5 & 11,1 & 1 & 4 & \\
\hline
\end{tabular}

*10 semestre $(n=68) ; 40$ semestre $(n=67) ; 8$ semestre $(n=64)$.

9,1\% ( $n=9)$ estavam na fase de alerta, 4\% $(n=4)$ na fase de quase exaustão e $1 \%(n=1)$ na fase de exaustão. Com relação à sintomatologia, $69,4 \%(n=68)$ desses alunos relataram predominância de sintomas psicológicos, seguidos de $20,4 \%(n=20)$ com sintomas físicos e de 10,2\% ( $n=10)$ com sintomas físicos e psicológicos concomitantes.

Observou-se, ainda, que 65,5\% ( $n=130)$ dos alunos não se sentiam bem repousados ao acordar pela manhã, embora $55,2 \%(n=110)$ considerassem a sua qualidade de sono melhor do que se possa imaginar. Verificou-se, também, que daqueles que informaram uma qualidade de sono pior do que se possa imaginar, $75,2 \%(n=64)$ apresentaram sintomas de estresse, assim como entre os alunos que relataram não se sentir bem repousados ao acordar pela manhã, 63\% ( $n=82)$ tinham sintomas de estresse. Embora uma grande proporção $(73,5 \%)$ tenha considerado como fonte de estresse as suas atividades acadêmicas, apenas 35,5\% $(n=71)$ dos estudantes informaram participar de alguma atividade para a promoção de saúde física e/ou mental. É importante notar que $71,7 \%(n=71)$ dos que não realizavam atividade para promoção de saúde apresentaram sintomas de estresse.

\section{DISCUSSÃO}

A prevalência de sintomas de estresse nos estudantes de Medicina, nesse estudo, foi de 49,7\% $(n=99)$. Os dados desse estudo mostraram maior prevalência percentual do sexo masculino $(54,5 \%)$ entre os estudantes de Medicina, embora os índices de sintomas de estresse tenham se revelado superiores no sexo feminino (66,7\%\% versus 35,8\%). Esses resultados são concordantes com os encontrados na popu- 
lação em estudos realizados com estudantes de Medicina de outras instituições, que evidenciam maior suscetibilidade das mulheres às fontes de estresse $e^{7,8,18}$.

Embora estudos apontem os fatores ocupacionais como responsáveis pelos distúrbios emocionais nos médicos, resultados de revisão indicam que a história de vida, os antecedentes pessoais e familiares e os aspectos de personalidade mostram-se mais significativos na gênese de tais transtornos nessa população ${ }^{3,4,19}$. Aspectos psicodinâmicos, como comportamento compulsivo, rigidez, atitude de controle das emoções, retardo de gratificações e fantasias irrealistas acerca do futuro, tanto influenciam a escolha pela carreira médica, quanto predispõem a distúrbios emocionais, abuso de álcool e drogas e doença mental ${ }^{4}$.

Infelizmente, não foi analisada a prevalência de abuso de álcool e drogas, por serem questões mais difíceis de ser analisadas com boa especificidade, bem como por não constituírem o foco da presente pesquisa.

Uma análise da ocorrência da sintomatologia de estresse no primeiro, quarto e oitavo semestres revela níveis mais elevados no $4^{\circ}$ semestre $(67,2 \%)$, concordantes com resultados de outros estudos ${ }^{20-22}$. Esse período corresponde ao momento em que o estudante inicia as disciplinas clínicas e, consequentemente, tem mais contato com o paciente, a doença e o sofrimento. É importante considerar que o estresse mostra-se presente nos três semestres avaliados, mas em diferentes níveis para cada período. $\mathrm{O}$ índice de sintomas de estresse no momento de ingresso na faculdade $(41,2 \%)$ pode ser porque este fato constitui uma fase de transição em relação ao estilo de vida, que exige um período de adaptação às diversas mudanças inerentes à vida acadêmica ${ }^{7,20,22,23}$. Além disso, o volume de informações a apreender, as limitações de tempo, as avaliações, a competição, as questões concernentes a relações íntimas, aos aspectos financeiros e familiares foram identificados como estressores relatados por estudantes de Medicina ${ }^{24-26}$. No $8^{\circ}$ semestre, a prevalência de sintomas de estresse $(40,6 \%)$ diminuiu em relação ao $4^{\circ}$ semestre $(67,2 \%)$. Embora aquele seja o momento em que o aluno prepara-se para a entrada no internato, essa diminuição poderia ser explicada pela adaptação do estudante ao meio e também por já ter ultrapassado a transição do Ciclo Básico para o Clínico.

Observou-se, ainda, a prevalência do estresse na fase de resistência em 85,8\% ( $n=85)$ dos estudantes. Isso pode indicar que os estudantes conseguem proteger-se até certo ponto dos estímulos estressores, pois apenas $4 \%(n=4)$ encontravam-se na fase de exaustão, e 1\% $(n=1)$ na fase de quase-exaustão. Ainda que uma pequena proporção tenha alcançado fases mais avançadas de estresse, vale ressaltar o significado da fase de resistência na saúde do indivíduo, pois a busca pela homeostase pode torná-lo vulnerável a infecções e doenças, tendo em vista a hiperatividade córtico-suprarrenal e um dispêndio excessivo de energia ${ }^{17}$.
Nesse estudo, apesar de 73,5\% ( $n=147)$ dos alunos considerarem suas atividades na faculdade como fonte de estresse, apenas 35,8\% ( $n=71)$ relataram participar de alguma atividade voltada à promoção de sua saúde física e/ou mental. Ademais, os resultados obtidos sobre a relação do estresse e a prática de atividade para promoção de saúde são coerentes com estudos que revelam a eficácia do exercício físico na redução de ansiedade e na melhora da autopercepção física e da autoestima ${ }^{27-29}$, evidenciando assim a importância dessa atitude na prevenção do estresse bem como o desconhecimento ou desinteresse do aluno por essas questões.

\section{CONCLUSÃO}

Este estudo mostrou níveis significativos de estresse nos estudantes de Medicina, especialmente no quarto semestre do curso, quando há o envolvimento clínico. Evidenciou-se, ainda, que, nos três semestres estudados, os alunos com sintomas de estresse encontravam-se, em sua maioria, na fase de resistência, bem como apresentavam predominância de sintomatologia na esfera psicológica. Observou-se também que as estudantes apresentaram maior prevalência de estresse em relação aos alunos do sexo masculino.

\section{REFERÊNCIAS}

1. Nogueira-Martins LA. A saúde do profissional de saúde. In: De Marco MA (ed.). A face humana da medicina: do modelo biomédico ao modelo biopsicossocial. São Paulo: Casa do Psicólogo; 2003.

2. Nogueira-Martins LA. Saúde mental dos profissionais de saúde. In: Botega NJ (ed.). Prática psiquiátrica no hospital geral: interconsulta e emergência. Porto Alegre: Artmed; 2002.

3. Nogueira-Martins LA. A saúde mental do médico e do estudante de medicina. Int J Psychiatry Med. 1996. (Acesso em: 18 de março de 2006). Disponível em: http://www. polbr.med.br/arquivo/saudment.htm.

4. Ramos-Cerqueira AT, Lima MC. A formação da identidade do médico: implicações para 0 ensino de graduação em Medicina. Interface - Comunic, Saúde, Educ. 2002;6(11):107-16.

5. Castro FC. Os temores na formação e prática da medicina: aspectos psicológicos. Rev Bras Educ Méd. 2004;28(1):38-45.

6. Dyrbye LN, Thomas MR, Shanafelt TD. Systematic review of depression, anxiety, and other indicators of psychological distress among U.S. and Canadian medical students. Acad Med. 2006;81(4).

7. Furtado E, Falcone E, Clark C. Avaliação do estresse e das habilidades sociais na experiência acadêmica de estudantes de medicina de uma Universidade do Rio de Janeiro. Interação em Psicologia. 2003;7(2):43-51.

8. Souza FGM, Menezes MGC. Estresse nos estudantes de medicina da Universidade Federal do Ceará. Rev Bras Educ Méd. 2005;29(2).

9. Zonta R, Robles ACC, Grosseman S. Estratégias de enfrentamento do estresse desenvolvidas por estudantes de medicina da Universidade Federal de Santa Catarina. Rev Bras Educ Méd. 2006;30(3)

10. Millan LR, Souza EN, Marco OLN, Rossi E, Arruda PCV. O I encontro paulista dos serviços de assistência psicológica ao estudante universitário. Rev Hosp Clin Fac S Paulo. 1998;53(3):156-61. 
11. Gouveia VV, Pinheiro AG. Valores e atitudes dos estudantes de Medicina. In: Pimentel AJP, Andrade E0, Barbosa GA (org.). Os estudantes de medicina e 0 ato médico. Brasília: ConseIho Federal de Medicina; 2004.

12. Morrison J, Moffat K. More on medical student stress. Med Educ. 2001;35:617-9.

13. Eizirik CL. 0 médico, o estudante de medicina e a morte. Rev AMRIGS. 2000;44(1/2):50-5.

14. Lipp MEN, Guevara AJH. Validação empírica do inventário de sintomas de stress (ISS). Estud Psicol. 1994;11(3):43-9.

15. Camelo SHH, Angerami ELS. Sintomas de estresse nos trabalhadores atuantes em cinco núcleos de saúde da família. Rev Latino-Am Enfermagem. 2004;12(1):14-21.

16. Cardoso CL, Loureiro SR, Nelson-Filho P. Pediatric dental treatment: manifestations of stress in patients, mothers and dental school students. Braz Oral Res. 2004;18(2):150-5.

17. Lipp MEN. Manual do Inventário de Sintomas de Stress para Adultos de Lipp (ISSL). São Paulo: Casa do Psicólogo; 2005.

18. Dahlin $\mathrm{M}$, Joneborg N, Runeson B. Stress and depression among medical students: a crosssectional study. Med Educ. 2005;39:594-604.

19. Kaplan HI, Sadock BJ, Grebb JA. 0 relacionamento médico-paciente e técnicas de entrevista. In: Kaplan HI, Sadock BJ, Grebb JA. Compêndio de psiquiatria. Porto Alegre: Artmed; 2003.

20. Moro A, Valle JB, Lima LP. Sintomas depressivos nos estudantes de medicina da universidade da região de Joinville (SC). Rev Bras Educ Méd. 2005;29(2):97-102.
21. Porcu M, Fritzen CV, Helber C. Sintomas depressivos nos estudantes de medicina da Universidade Estadual de Maringá. Psiq Prát Méd. 2001;34(1).

22. Supe AN. A study of stress in medical students at seth g. s. medical college. J Postgrad Med. 1998;44:1-6.

23. Radcliffe C, Lester H. Perceived stress during undergraduate medical training: a qualitative study. Medl Educ. 2003;37:32-8.

24. Notman MT, Salt P, Nadelson CC. Stress and adaptation in medical students: who is most vulnerable? Compr Psychiatry. 1984;25:355-66.

25. Toews JA, Lockyer JM, Dobson DJ, Simpson E, Brownell AK, Brenneis F, et al. Analysis of stress levels among medical students, residents and graduate students at four Canadian schools of medicine. Acad Med. 1997;72(11):997-1002.

26. Vitaliano PP, Russo J, Carr JE, Heerwagen JH. Medical school pressures and their relationship to anxiety. J Nerv Ment Dis. 1984;172:730-6.

27. Camacho TC, Roberts RE, Lazarus NB, Kaplan GA, Cohen RD. Physical activity and depression: evidence from the alameda county study. Am J Epidemiol. 1991;134(2):220-31.

28. Fox KR. The influence of physical activity on mental well-being. Public Health Nutrition. 1999;2:411-8.

29. Harris AH, Cronkite R, Moos R. Physical activity, exercise coping, and depression in a 10jear cohort study of depressed patients. J Affect Disord. 2006;93(1/3):79-85. 